\title{
Multiplexing a serial array of tapered optical fibre sensors using coherent optical frequency domain reflectometry.
}

\author{
Renata Jarzebinska, Edmon Chehura, Stephen W James and Ralph P Tatam \\ Department of Engineering Photonics \\ School of Engineering \\ Cranfield University \\ Cranfield \\ Bedfordshire \\ UK \\ MK43 0AL
}

\begin{abstract}
The use of high spatial resolution optical frequency domain reflectometry to facilitate the multiplexing of a serial array of tapered optical fibre sensors is presented. Changes in the attenuation of the Rayleigh backscattered signal from the tapered regions are used to monitor the refractive index of the surrounding medium. The use of an array of 5 tapered regions to monitor liquid flow is demonstrated. An analysis of the performance of the system shows that, for tapers of diameter $50 \mu \mathrm{m}$, up to 9 tapers could be multiplexed using commercial optical frequency domain reflectometry (OFDR) instrumentation.
\end{abstract}

\section{Introduction.}

Optical fibre tapers offer a simple method for facilitating a strong interaction between the light propagating in the fibre and the surrounding environment, which has been exploited for evanescent wave spectroscopy ${ }^{1}$, for the measurement of refractive index of the surrounding medium ${ }^{2}$, and for chemical sensing by measuring chemically induced changes in the refractive index of functional coatings $^{3}$. In common with other fibre optic sensor (FOS) approaches that rely upon the measurement of changes in fibre attenuation resulting from an interaction between the measurand and the propagating light, difficulties associated with the multiplexing of serial arrays of sensors, and the requirement to work in transmission, can limit their practical application.

Among the techniques that may be used to interrogate a serial array of such sensors, the use of optical time domain reflectometry (OTDR) for monitoring such sensors is compromised by the limited spatial resolution of OTDR and the short gauge length of tapers. The multiplexing of an array of tapered fibre sensors has been demonstrated by combining the taper array with an array of wavelength division multiplexed fibre Bragg gratings, where an FBG was located after each tapered section ${ }^{4}$. In the configuration presented, the sensors were shown to provide an on-off response, where the Bragg peak of all FBGs that followed a particular tapered section would disappear from the spectrum as a result of the increase attenuation experienced when the taper is surrounded by a medium of refractive index higher than that of the cladding. This was used to monitor the flow of epoxy resin into a mould, but has limitations as the flow should follow the optical fibre, in the direction from the last taper to the first.

Coherent optical frequency domain reflectometry (OFDR) has been shown to be capable to identifying changes in attenuation of an optical fibre with high spatial resolution, and instrumentation is available commercially. OFDR is a distributed measurement technique that offers the ability to monitor the power of light backscattered along the length of an optical fibre under test, which might be as a result of Rayleigh scattering or of reflections from splices and components such as fibre Bragg gratings, with very high spatial resolution ${ }^{5,6,7,8}$. The basis of OFDR is swept wavelength homodyne interferometry, in 
which the output from a tuneable laser is input to an interferometer with one path containing the fibre under test and the other to a reference arm of fixed length. As the laser wavelength is tuned, each scattering location along the fibre creates a unique beat signal when mixed with the reference beam, the frequency being dependent on the path length imbalance. Fourier processing of the output of the interferometer allows the characterisation of the amplitude and phase of the reflections as a function of time, which, through knowledge of the effective index of the propagating mode, can be related to position along the fibre. This approach has been used in the characterisation of optical fibre modules and components and has been applied to the sensing of strain, by monitoring changes in the Rayleigh scattering of the fibre ${ }^{9,10,11,12}$ and is used in biomedical imaging ${ }^{13,14}$. Commercial instrumentation is capable of micron scale resolution with measurement ranges of order $100 \mathrm{~m}^{15}$.

Tapering an optical fibre allows the evanescent field of the propagating mode to penetrate into the surrounding environment, with the proportion of the power in the evanescent field increasing with decreasing diameter of the tapered region. This is accompanied by a local increase in attenuation of the fibre, which is sensitive to the refractive index of the surrounding medium, and which can be measured using OFDR. To date we are not aware of the use of OFDR to facilitate the demodulation and multiplexing of discrete sensor elements whose operation principle is based upon changes in attenuation. In this paper we explore the use of OFDR to multiplex an array of optical fibre taper sensors, and demonstrate their use to monitor liquid flow. The ability to monitor the flow of a liquid in this manner has applications in process monitoring during the fabrication of fibre reinforced composite materials, by monitoring the infusion of resin into a carbon or glass fibre composite layup $4^{16,17}$.

\section{Experimental}

\subsection{Optical fibre tapers - fabrication}

Optical fibre tapers were fabricated using the heat and draw approach in single mode optical fibre (Optical Fibres, SM-1322) with a cut off wavelength of $1322 \mathrm{~nm}$. The heat source used was a gas burner containing a butane $(65 \%) /$ propane $(35 \%)$ mixture, which was equipped with a nozzle enabling manual flame control. The size of the flame was $\sim 10 \mathrm{~mm}$ and the flame temperature $\sim 1970^{\circ} \mathrm{C}$. Prior to tapering, the buffer coating was removed from a $35 \mathrm{~mm}$ length of fibre using mechanical strippers. During the tapering process the ends of the fibre were attached to rotation stages which were set to rotate in opposite directions to pull the fibre with a constant velocity of $100 \mu \mathrm{m} \mathrm{s}^{-1}$. Photographs of the tapered fibre are shown in figure 1(a-b).

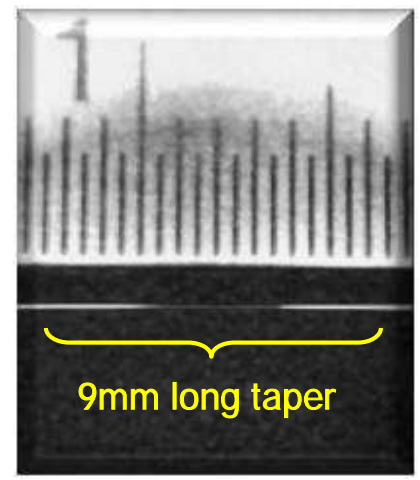

(a)

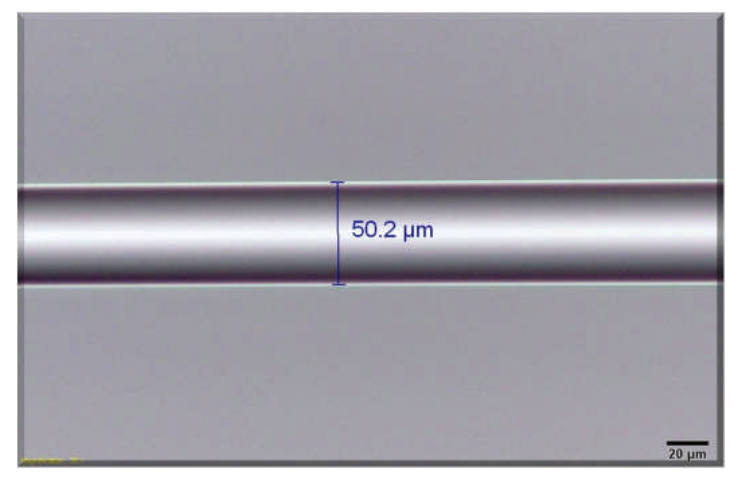

(b)

Figure. 1. Image of a tapered fibre; (a) image of the whole tapered region of a fibre, total taper's length-9.0mm; (b) image of the central section of the taper waist, the length of the region of uniform waist diameter is $\sim 2.6 \mathrm{~mm}$ recorded using an optical microscope. 


\subsection{Optical fibre tapers - characterisation}

A Luna Technologies' Optical Backscatter Reflectometer (OBR) was used to characterise the tapered optical fibres. The OBR allows high resolution characterisation of the Rayleigh scattering along the length of the optical fibre using the OFDR approach described in the introduction. A plot of the backscattered signal from an optical fibre containing a biconical taper of diameter $(51.2 \pm 0.5) \mu \mathrm{m}$, together with the experimental arrangement, is shown in figure 2. The amplitude of the Rayleigh backscatter signal obtained from a taper is characterised by a sharp decrease in the tapered region, and there is a residual decrease in the backscattered amplitude from the fibre after the taper as shown in figure 2(b). It should be noted that all of the plots of the Rayleigh backscatter signals are presented as the normalised amplitude of the backscattered signal, with unit $\mathrm{dB} / \mathrm{mm}$, plotted as a function of position along the optical fibre, the standard format provided by the OBR software. 




Figure 2(a). Rayleigh backscatter trace obtained from an optical fibre containing a (51.2 \pm 0.5$) \mu m$ diameter tapered region, with the sources of the reflection peaks indicated on the diagram of the experimental setup.

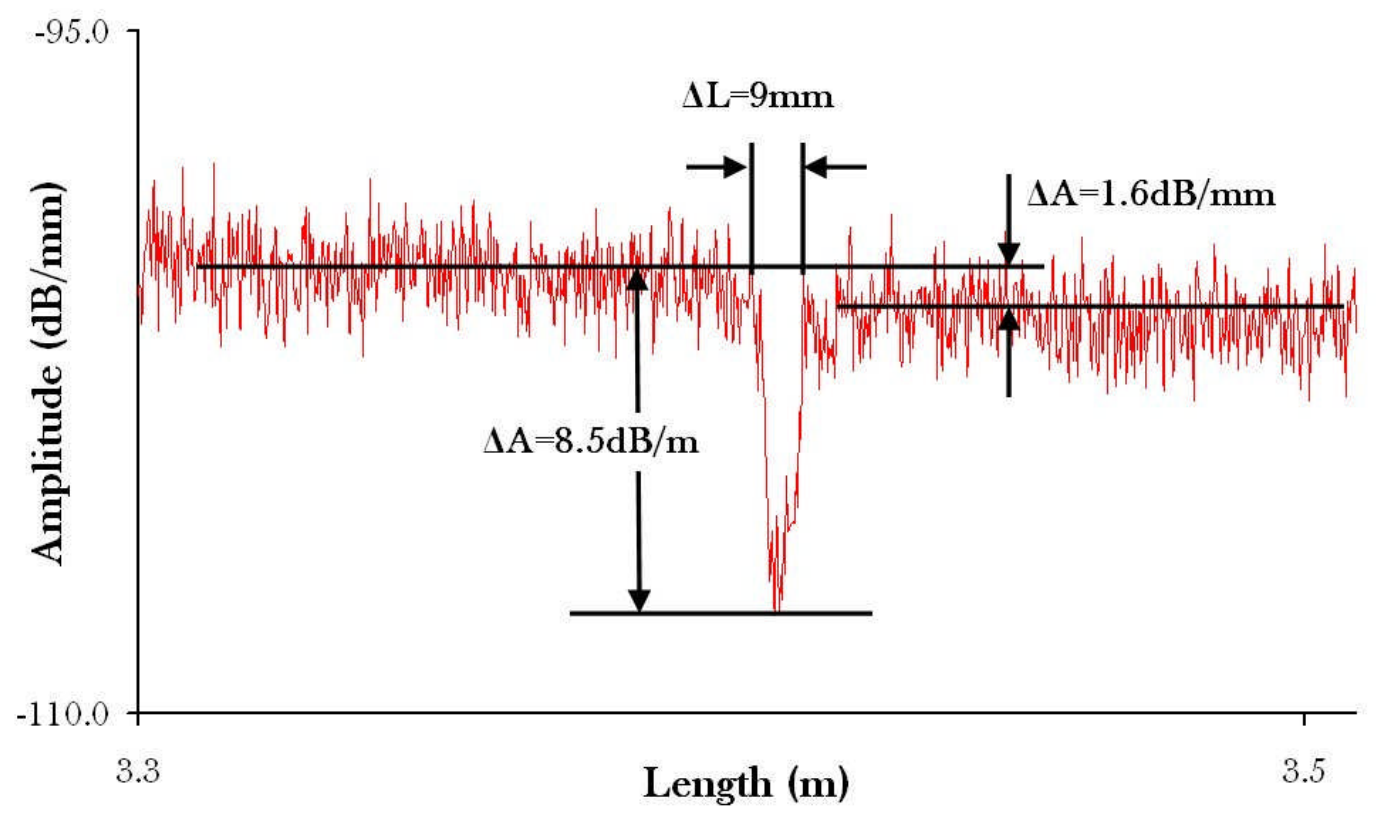

Figure 2(b). Rayleigh backscatter trace of an optical fibre containing a $(51.2 \pm 0.5) \mu m$ diameter tapered region (with the total taper length of $9 \mathrm{~mm}$ ) depicted in Figure 2(a), zoomed in on the tapered section.

The total length of the taper (waist and contracting zones on both sides) is $9 \mathrm{~mm}$ and the length of the section with uniform waist diameter is $2.6 \mathrm{~mm}$. The loss introduced by the presence of the taper is approximately $8.5 \mathrm{~dB} / \mathrm{mm}$ when the taper is surrounded by air. Figure 3(a) shows the response of the tapered region to immersion in Cargille oils of refractive indices in the range 1.4 to 1.7. The length of 
fibre from which the coating was removed was immersed in the oil, fully encompassing the tapered section. For clarity of the presentation the traces have been vertically offset. When there is a large refractive index contrast between the fibre and the oil, for example for oil indices of 1.4 and 1.5, the taper is characterized by a decrease in Rayleigh backscatter amplitude, while for surrounding refractive indices that produce a low contrast the backscatter signal from the tapered section increases. For surrounding refractive indices greater than or equal to the cladding refractive index, the backscattered signal also contains a peak originating outside the tapered section, with its amplitude and the distance from the centre of the tapered region decreasing with increasing refractive index. The physical origins of these features are unclear and are the subject of further investigation. It is suggested that the increase in the measured backscattered signal within the tapered region is a result of an increase in the efficiency with which the Rayleigh scattered light is coupled into the modes of the taper, facilitated by surrounding refractive index induced changes in the electric field profile of the modes. For the purpose of this paper, the key feature is the residual decrease in the backscattered signal after the tapered section, which is plotted in Figure 3(b), showing peak sensitivity at 1.456 RIU. 


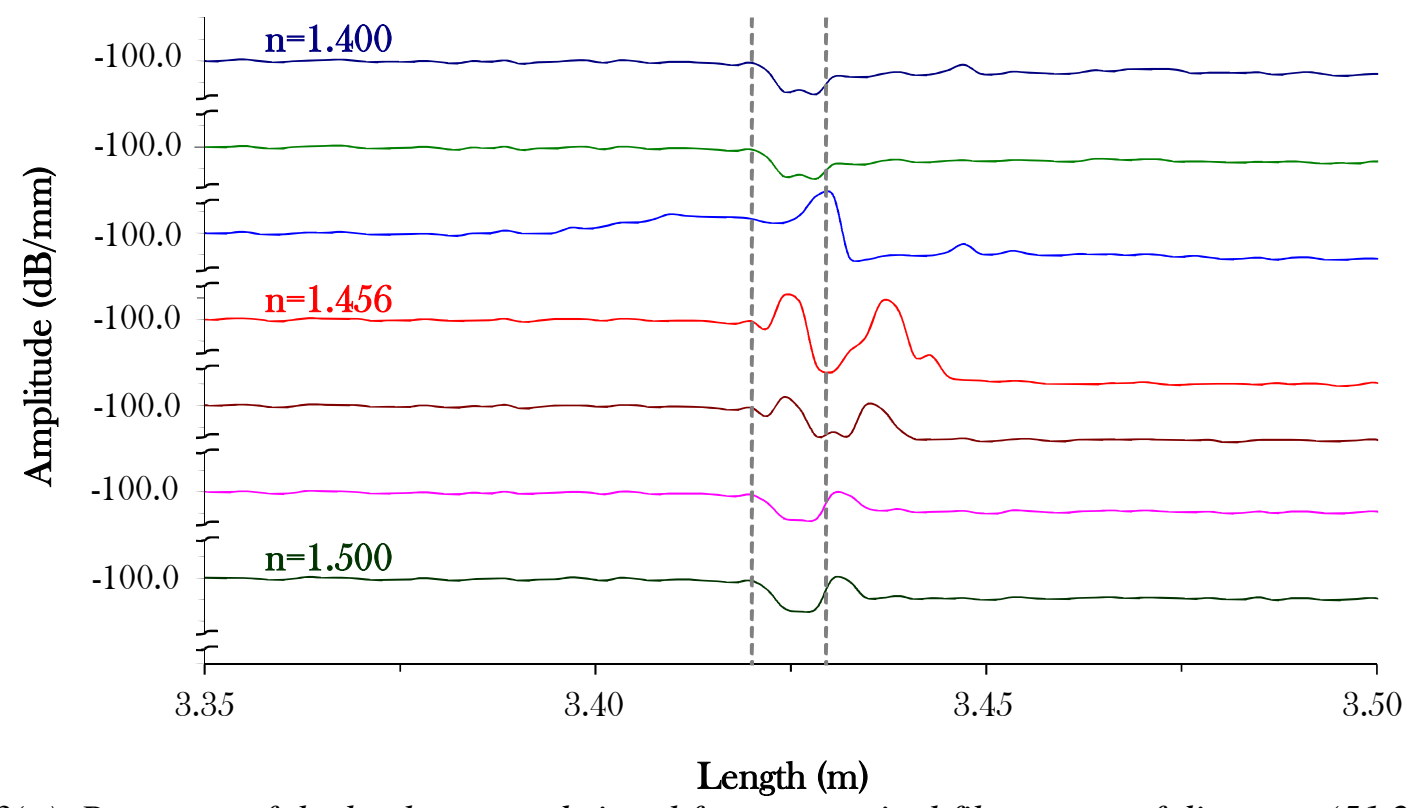

Figure 3(a). Response of the backscattered signal from an optical fibre taper of diameter (51.2 \pm 0.5$) \mu m$ to immersion in oils of differing refractive index; one division on the y-axis represents $5 \mathrm{~dB} / \mathrm{mm}$. The dotted lines indicate the extent of the tapered region of the optical fibre.



Figure 3(b). Plot of the loss introduced by the (51.2 \pm 0.5$) \mu m$ taper as a function of surrounding refractive index. The line is a guide to the eye. Measurands' uncertainties are included in the graph.

The response is similar to that reported previously for a range of fibre devices, e.g. side-polished optical fibres ${ }^{18}$ and long period gratings ${ }^{19}$. The loss introduced by the taper as a function of surrounding refractive index was calculated as the difference between the average signal amplitudes in the sections of fibre immediately before and after the taper.

\section{Multiplexing - Results and Discussion}


With the aim of investigating the multiplexing of a serial array of tapered optical fibre sensors, an array of five tapers was fabricated. The tapered regions had diameters of $(51.2 \pm 0.5) \mu \mathrm{m},(50.2 \pm 0.4) \mu \mathrm{m}$, $(50.4 \pm 0.4) \mu \mathrm{m},(49.5 \pm 0.7) \mu \mathrm{m}$ and $(50.1 \pm 0.4) \mu \mathrm{m}$, and adjacent tapers were separated physically by $\sim 140$ $\mathrm{mm}$. The evolution of the backscattered trace as the tapered sections were fabricated is described in this section.

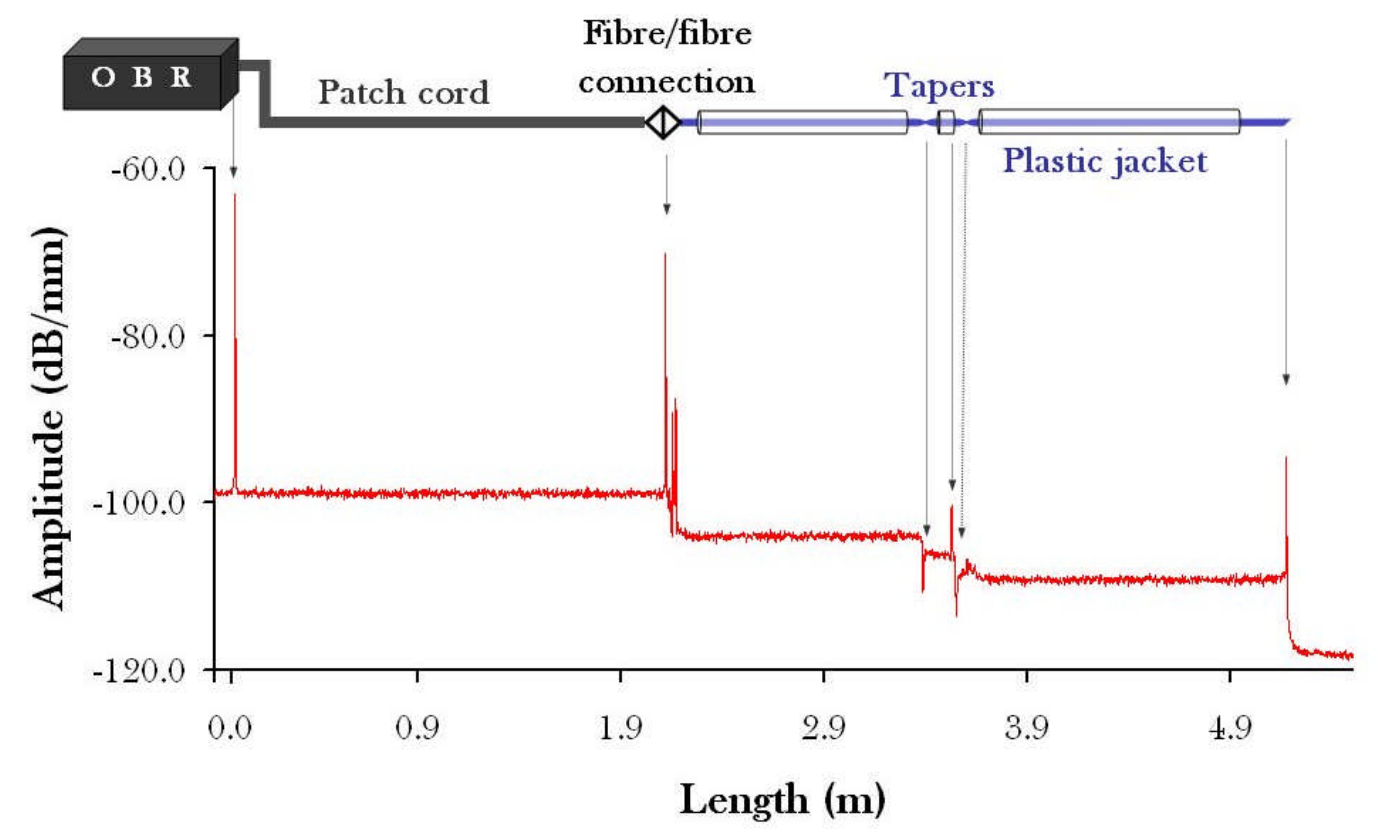

Figure 4(a). Rayleigh backscatter trace obtained from an optical fibre containing tapered sections of diameter $(51.2 \pm 0.5) \mu \mathrm{m}$ and $(50.2 \pm 0.4) \mu \mathrm{m}$, with the sources of the reflection peaks indicated on the diagram of the experimental setup above.

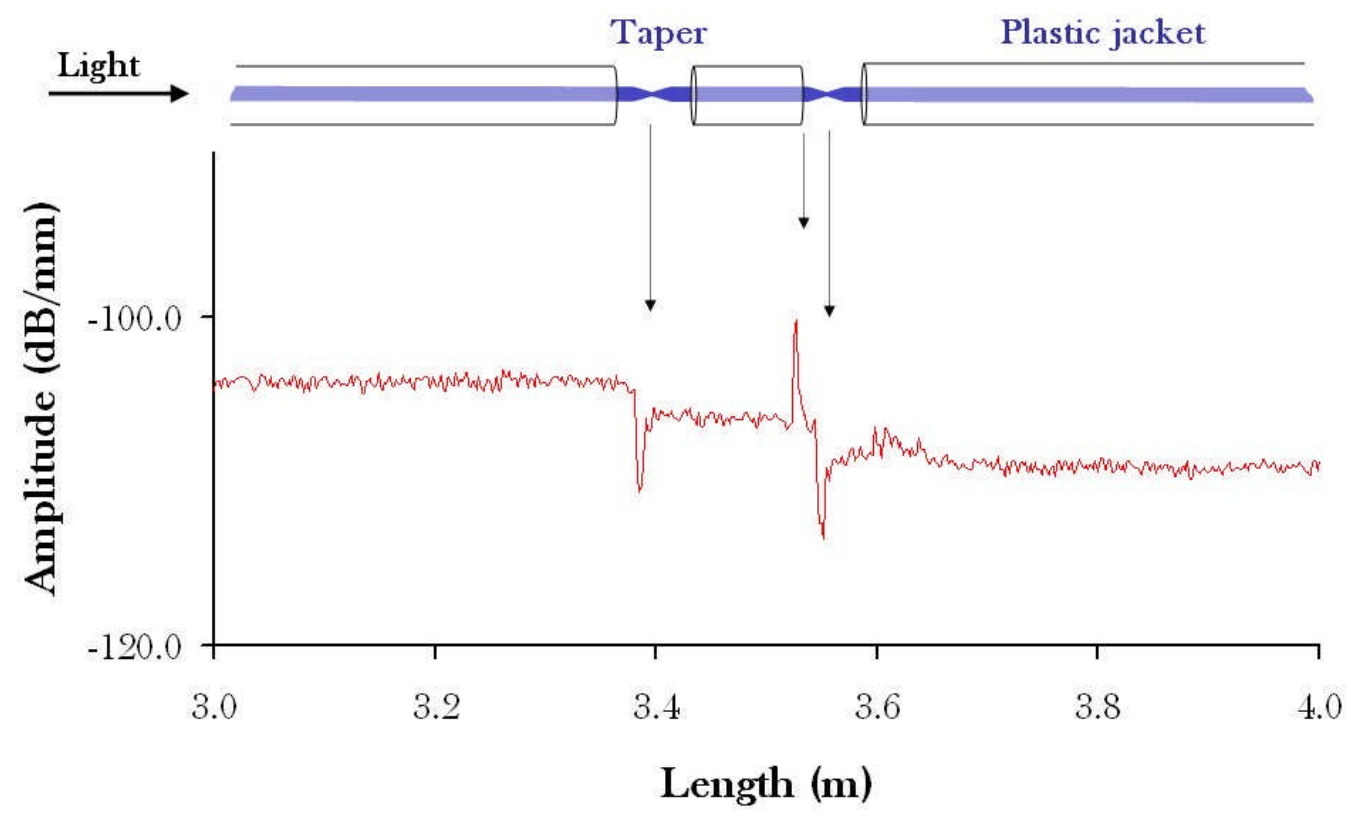

Figure 4(b). Rayleigh backscatter trace obtained from an optical fibre containing tapered sections of diameter $(51.2 \pm 0.5) \mu \mathrm{m}$ and $(50.2 \pm 0.4) \mu \mathrm{m}$, zoomed on the tapered sections. 
Figure 4(a-b) shows the signal obtained from an optical fibre containing two tapered regions. The fabrication of the second taper introduces a second region of attenuation, which is preceded by a peak. The peak is located at the end of the buffer coating before the stripped region of fibre containing the second taper. It is likely that the discontinuity in the effective index of the cladding modes at the boundary between the sections of fibre with and without the buffer coating produces the observed reflection peak. This was confirmed by stripping the buffer jacket from a subsequent section of the fibre, as illustrated in figure 5. At the output from the tapered region, it is likely that light will be coupled into cladding modes of the fibre which, within the subsequent buffer-coated region of the fibre, would be coupled into the coating, and could propagate in whispering gallery modes ${ }^{20}$. The whispering gallery modes would be partially reflected at the next discontinuity in the coating, producing the peak observed in the results. It is known that whispering gallery modes can recouple to the core mode, as this has been shown to influence bend loss measurements ${ }^{21}$. 


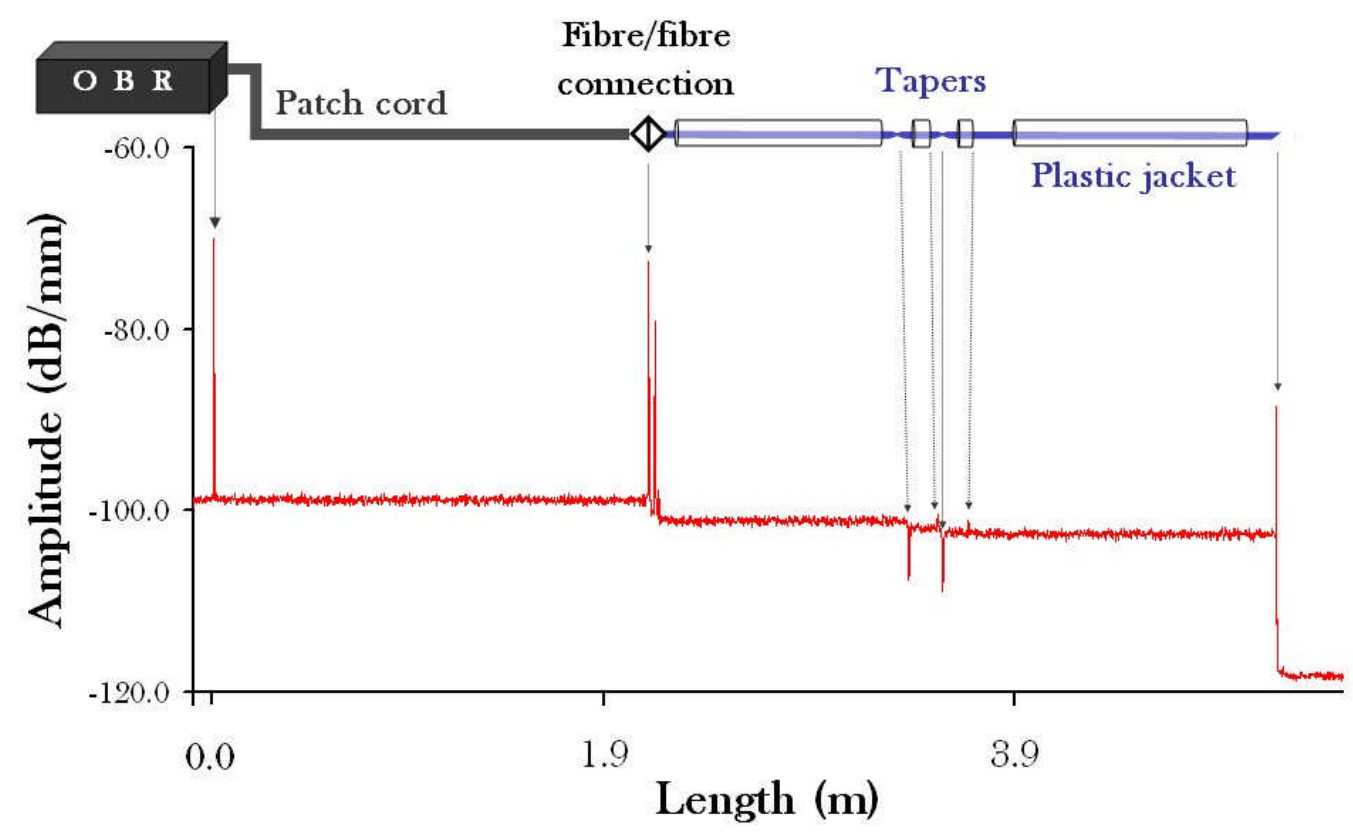

Figure 5(a). Rayleigh backscatter trace obtained from an optical fibre containing two tapered sections of diameter (51.2 \pm 0.5$) \mu \mathrm{m}$ and (50.2 \pm 0.4$) \mu \mathrm{m}$ respectively, with a section of fibre stripped of its buffer jacket, located $14.0 \mathrm{~mm}$ away from the second taper and with the sources of the reflection peaks indicated on the diagram of the experimental setup above.

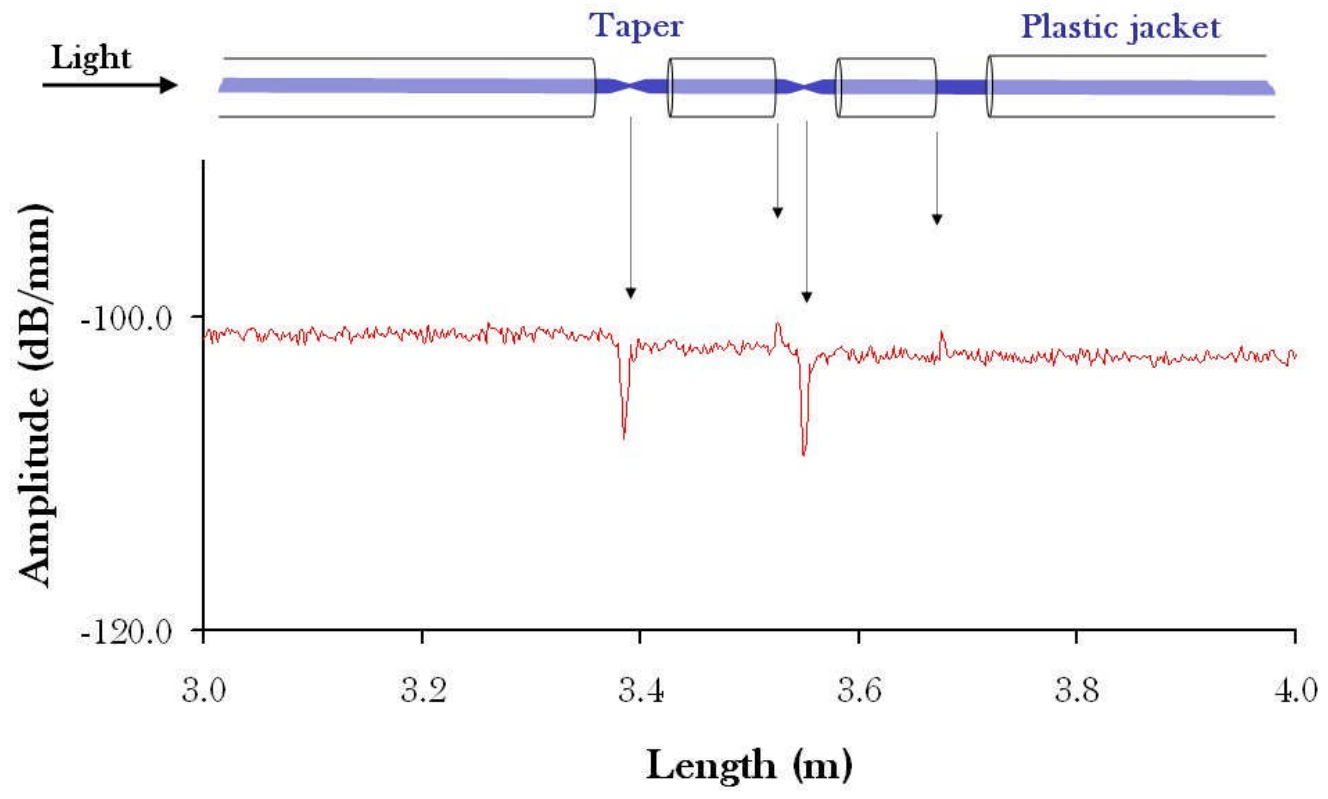

(b)

Figure 5(b). Rayleigh backscatter trace obtained from an optical fibre containing two tapered sections of diameter (51.2 \pm 0.5$) \mu m$ and (50.2 \pm 0.4$) \mu m$ respectively, with a section of fibre stripped of its buffer jacket, located $14.0 \mathrm{~mm}$ away from the second taper, zoomed on the tapered sections.

Figure 6 shows the backscatter trace observed following the fabrication of an array of five tapers, of diameters $(51.2 \pm 0.5) \mu \mathrm{m},(50.2 \pm 0.4) \mu \mathrm{m},(50.4 \pm 0.4) \mu \mathrm{m},(49.5 \pm 0.7) \mu \mathrm{m}$ and $(50.1 \pm 0.4) \mu \mathrm{m}$ respectively. Following the first tapered region, each of the subsequent tapered sections are characterised by the 
refection from the end of the buffer coating, a sharp increase in loss within the tapered region, and a small residual loss after the tapered section.

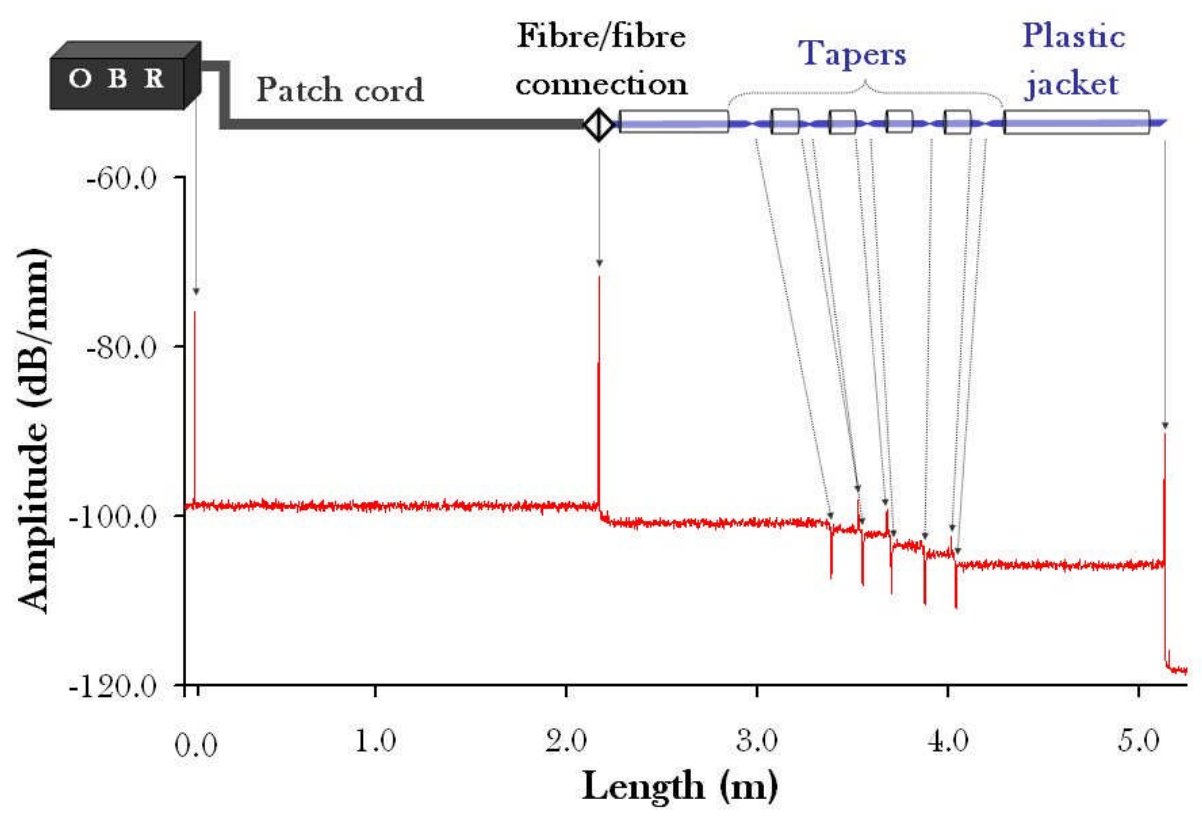

Figure 6(a). Rayleigh backscatter trace obtained from an optical fibre containing five tapers of diameters (51.2 \pm 0.5$) \mu \mathrm{m},(50.2 \pm 0.4) \mu \mathrm{m},(50.4 \pm 0.4) \mu \mathrm{m},(49.5 \pm 0.7) \mu \mathrm{m}$ and (50.1 \pm 0.4$) \mu \mathrm{m}$, with the sources of the reflection peaks indicated on the diagram of the experimental setup above.

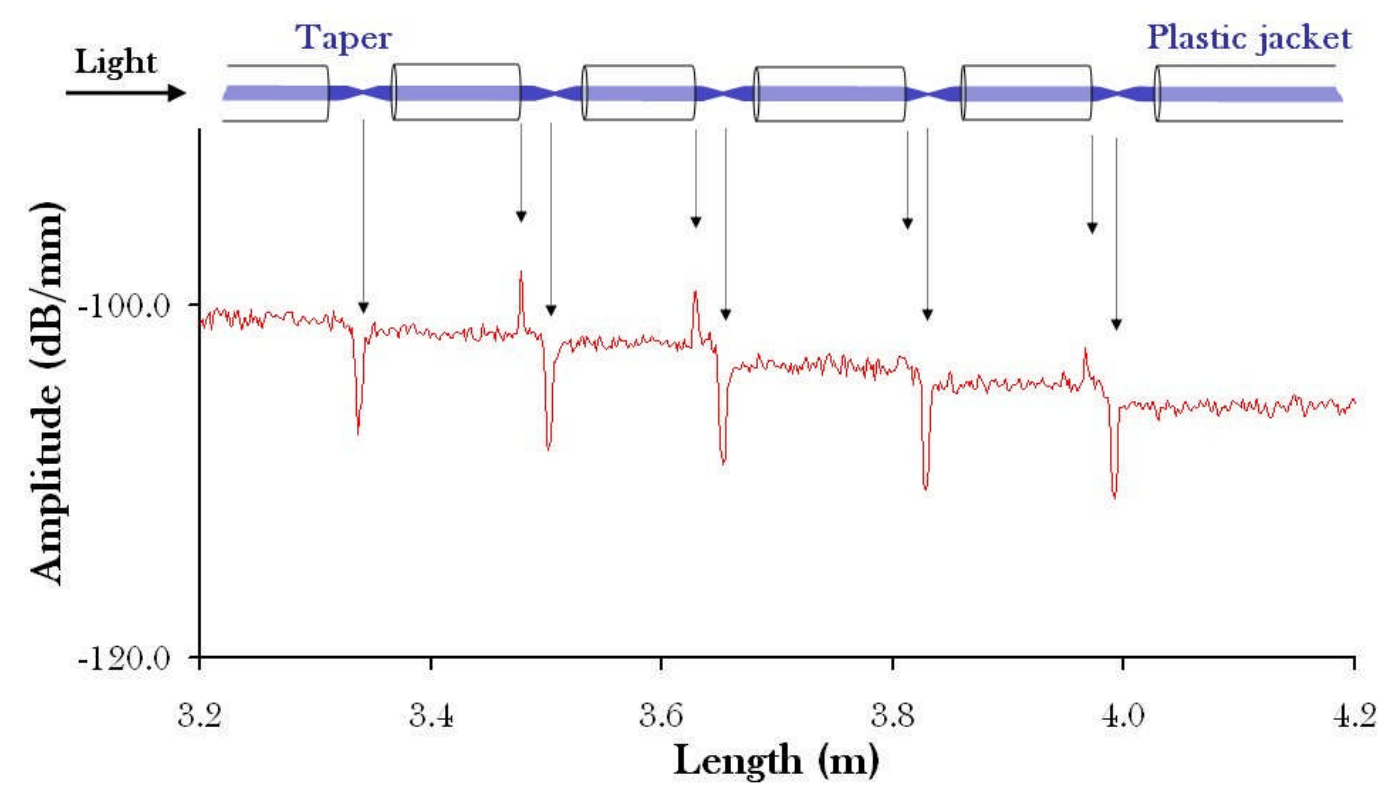

Figure 6(b). Rayleigh backscatter trace obtained from an optical fibre containing five tapers of diameters (51.2 \pm 0.5$) \mu \mathrm{m},(50.2 \pm 0.4) \mu \mathrm{m},(50.4 \pm 0.4) \mu \mathrm{m},(49.5 \pm 0.7) \mu \mathrm{m}$ and (50.1 \pm 0.4$) \mu \mathrm{m} ; \mathrm{zoom}$ on the section with tapers.

To demonstrate the use of the array of tapered optical fibres for monitoring the flow of a fluid, the optical fibre was mounted on a table and drops of oil of refractive index 1.52, representative of the refractive index of resins used to form the matrix in fibre reinforced composites, were placed onto the 
fibre in subsequent steps, imitating unidirectional oil flow. This way all of the tapers and the intermediate buffer jacketed regions of the optical fibre were sequentially immersed in oil, starting from the tapered section closest to the light source. The backscattered signal was monitored using the OBR, as indicated in figure 7.

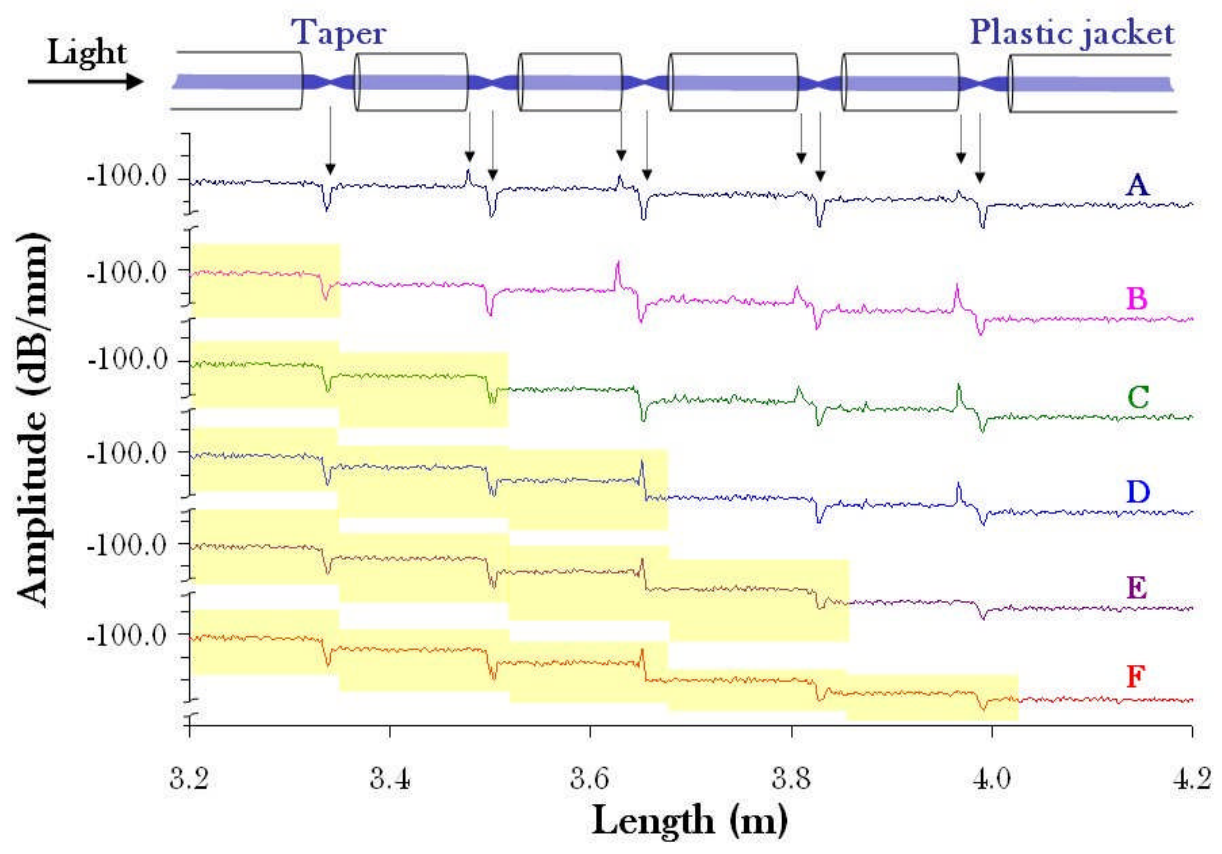

Figure 7. Evolution of the backscattered signal recorded as the oil flowed over the array of five optical fibre tapers. The amplitude signal was vertically offset for the clarity of the graph. The yellow shaded region provided a representation of the location of the oil as it reached subsequent tapers shown in signals labelled from $B$ to $F$; signal A was recorded with no oil applied. One division on the y-axis represents $5 \mathrm{~dB} / \mathrm{mm}$.

No change in the backscattered signal was observed as the oil flowed over the untapered, buffer jacketed, regions of the fibre. When the oil covered a taper, the residual attenuation of the backscattered signal from the subsequent sections of fibre increased, producing a step-like change in the backscattered amplitude. To demonstrate that the flow does not have to follow the fibre, encountering the tapered sections sequentially, figure 8 shows the backscatter signal obtained when taper 1 together with 4 , and 3 together with 5 , were covered by the oil. This investigation has demonstrated the potential capability of the technique to monitor resin infusion during the production of composite components, where the presence of resin in critical areas of a component is often a key requirement. Resin flow in such components, particularly those of complex shape, is often complicated compared to the primarily unidirectional flow in the resin transfer moulding (RTM) process used in the fabrication of predominantly planar components. 


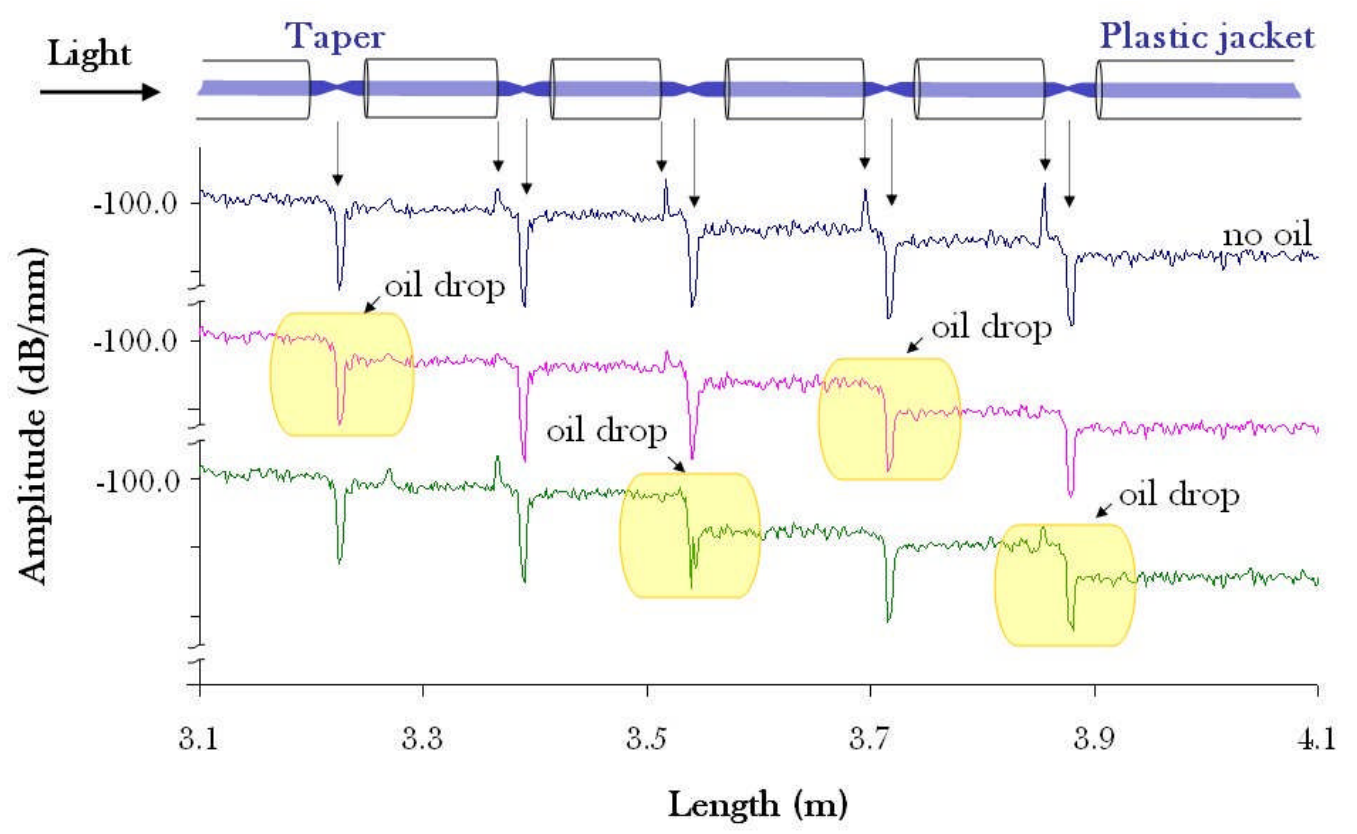

Figure 8. Evolution of the backscattered signal recorded as the oil encounters randomly chosen tapered sections of the fibre. The amplitude signal was vertically offset for the clarity of the graph. The yellow shaded region provided a representation of the location of the oil drops; one division on the $y$ axis represents $5 \mathrm{~dB} / \mathrm{mm}$.

It is informative to consider the number of tapered regions that could be multiplexed. With this in mind, the dependence of the change in residual attenuation on the taper diameter when the taper was immersed in oil of RI 1.52 was characterised. The results are plotted in figure 9 . The average signal loss induced by each taper is $\sim 2 \mathrm{~dB} / \mathrm{mm}$. The OBR has a noise floor of $\sim 118 \mathrm{~dB} / \mathrm{mm}$ allowing about 9 sensors to be multiplexed within a single length of fibre. The use of tapers with larger diameter, which would introduce a smaller decrease in the amplitude loss when immersed in the oil, would allow the multiplexing of a larger number of tapered sections but with lower sensitivity to the change in the surrounding RI for each sensor.

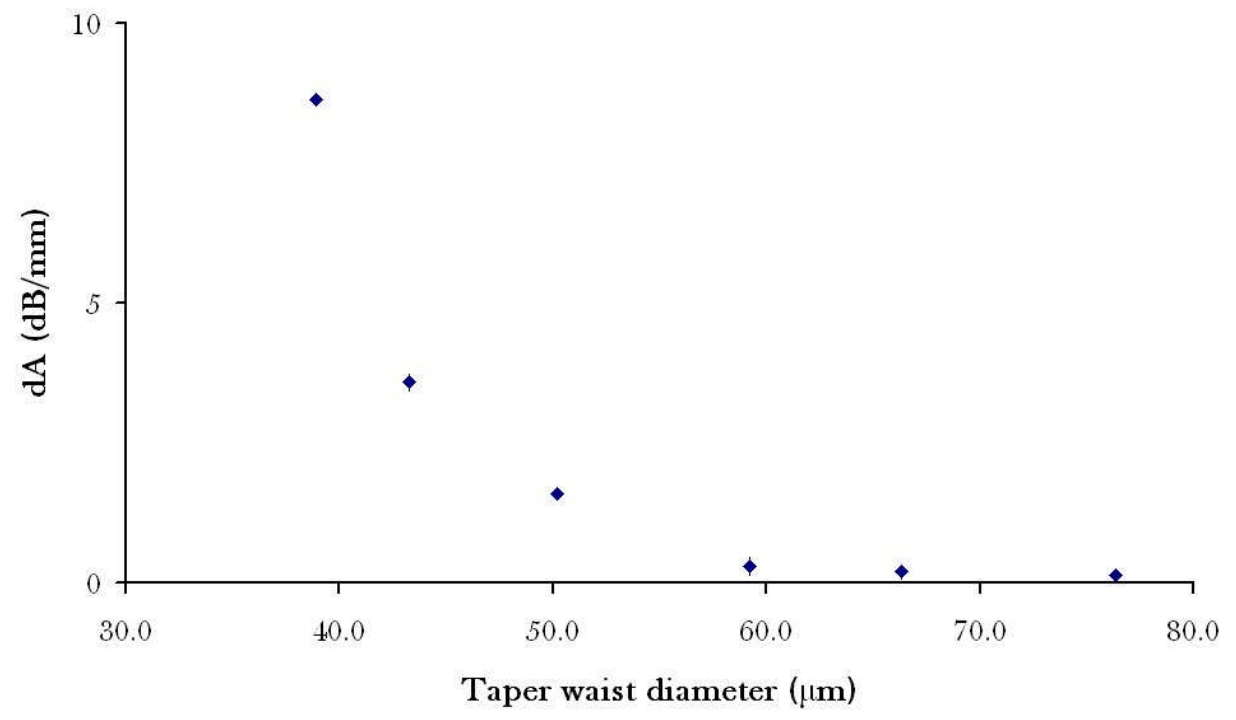

Figure 9. Backscattered signal amplitude loss/drop (dA) for different taper diameters upon immersion in oil of refractive index 1.52. The measurands' uncertainties are included in the graph. 
Fig. 9 indicates that the largest taper diameter for which it is possible to measure a change in attenuation due to the presence of a liquid of refractive index larger than that of the cladding is $\sim 60 \mu \mathrm{m}$. This is useful information as most resins that are in commercial use in the processing of carbon fibre reinforced composite components and structures for aerospace applications have refractive indices that are significantly larger than that of the optical fibre.

\section{Conclusions}

Coherent optical frequency domain reflectometry has been used to interrogate a tapered optical fibre, allowing characterization of the refractive index sensitivity of the loss of the tapered fibre section. The ability of OFDR to measure the Rayleigh backscattered signal from the fibre with high spatial resolution has been used to demonstrate a liquid flow sensor based on a multiplexed array of tapers fabricated within a single length of optical fibre. The approach allows determination of the time at which the liquid reaches a tapered section, which may have application in the monitoring of the infusion of resin into a fibre reinforced composite layup.

\section{Acknowledgments}

This work was partially supported by the Engineering and Physical Research Council (EPSRC), UK (EP/H0225X/1) and the European Commission Seventh Framework Programme via the Advanced Integrated Tail Cone (ADVITAC) programme (EU FP7-AAT-2008-RTD-1-234290).

\section{References}

[1] Paul P H and Kychakoff G 1987 Fiber-optic evanescent field absorption sensor Appl. Phys. Lett., 51 $12-14$

[2] Kumar A, Subrahmanyam T V B, Sharma A D, Thyagarajan K, Pal B P, and Goyal I C 1984 Novel Refractometer Using A Tapered Optical Fibre”. Electron. Lett. 20 534-535

[3] Jarzebinska R, Cheung C S, James S W, Tatam R P 2009 Response of the transmission spectrum of tapered optical fibres to the deposition of a nanostructured coating Meas. Sci. Technol. 20034001

[4] Gupta N and Sundaram R 2009 Fiber optic sensors for monitoring flow in vacuum enhanced resin infusion technology (VERITy) process Composites: Part A 40 1065-1070

[5] Glombitza U and Brinkmeyer E 1993 Coherent frequency domain reflectometry for characterization of single-mode integrated optical waveguides J. Lightwave Technol. 11 1377-1384

[6] Eickhoff W and Ulrich R, Optical frequency domain reflectometry in single-mode fiber $1981 \mathrm{Appl}$. Phys. Lett. 39, 693-695

[7] Soller B, Gifford D, Wolfe M and Froggatt M 2005 High resolution optical frequency domain reflectometry for characterization of components and assemblies Opt. Express 13, 666-674

[8] Chehura E, James S W and R P Tatam R P 2010 A simple and wavelength-flexible procedure for fabricating phase-shifted fibre Bragg gratings, Meas. Sci. Technol. 21, 94001

[9] Sang A K, Froggatt M E, Gifford D K, Kreger S T and Dickerson B D 2008 One centimeter spatial resolution temperature measurements in a nuclear reactor using Rayleigh scatter in optical fiber IEEE Sensors J. 8 1375-1380

[10] Imahama M, Koyamada Y and Hogari K 2008 Restorability of Rayleigh backscatter traces measured by coherent OTDR with precisely frequency-controlled light source IEICE Transactions on Communications E91-B, 1243-1246 
[11] Gifford D K, Sang A K, Kreger S T, Froggatt M E 2010 Strain measurements of a fiber loop rosette using high spatial esolution Rayleigh Scatter distributed sensing Proc. SPIE 7653, 765333

[12] Maier R R J, MacPherson W N, Barton J S, McCulloch S and Jones J D C 2009, Distributed sensing using Rayleigh scatter in polarization-maintaining fibres for transverse load sensing Meas. Sci. Technol. 21094019.

[13] Choma M A, Sarunic M V, Yang C H and Izatt J A 2003 Sensitivity advantage of swept source and Fourier domain optical coherence tomography Opt. Express 11, 2183-2189

[14] Ford H D and Tatam R P 2011 Characterisation of optical fibre imaging bundles for swept-source OCT Appl. Opt. 50, 627-640

[15] http://www.lunatechnologies.com/products/obr/obr_main.html (accessed 11/01/2012)

[16] Kueh S R M, Parnas R S, Dunkers J P, Advani G S, Paige S, Furrows M, Jones E, and Bailey T A 2000 Long Period Gratings As Flow Sensors For Liquid Composite Molding Proc. SPIE 3993, 240250

[17] Skordos A A, Karkanas P I and Partridge I K 2000 A dielectric sensor for measuring flow in resin transfer moulding, Meas. Sci. Technol. 11, 25-31

[18] Charters R B 1995 Continuous fibre optic components utilising evanescent coupling to organic thin films, PhD Thesis, Cranfield University

[19] Khaliq S, James S W and Tatam R P 2001 Fibre optic liquid level sensor using a long period grating, Opt. Lett. 26 , 1224-1226

[20] Morgan R D, Jones J D C, Barton J S and Harper P G 1994 Determination of monomode fiber buffer properties J. Lightwave Technol. 12, 1355-1359

[21] Morgan R D, Barton J S, Harper P G and Jones J D C 1990 Wavelength dependence of bend loss in monomode optical fibers: effect of the fiber buffer coating Opt. Lett. 15, 941 